\title{
A Combined Surgical Approach for Extensor Hallucis Longus Reconstruction: Two Case Reports
}

\author{
Marta Duarte ${ }^{1}$ Nuno Fradinho ${ }^{1}$ \\ ${ }^{1}$ Department of Plastic Reconstructive and Aesthetic Surgery, \\ Central Lisbon Hospital Centre, Lisbon, Portugal
}

Indian J Plast Surg 2021;54:225-228.

\begin{abstract}
Address for correspondence Marta Duarte, Department of Plastic Reconstructive and Aesthetic Surgery, Central Lisbon Hospital Centre, R. José António Serrano, 1150-199 Lisbon, Portugal (e-mail: martaduartek@gmail.com).
\end{abstract}

\begin{abstract}
The surgical management of foot tendon injuries is not well-represented in literature. To achieve excellent functional recovery of the extensor hallucis longus (EHL) tendon, we aimed at developing a reliable and feasible reconstructive technique.

A surgical technique for delayed reconstruction of the EHL tendon, combining an elongation procedure with second toe extensor tendon transfer, is described in this article.

Keywords

- extensor hallucis longus

- tendon

- reconstruction The results of this combined approach for EHL tendon reconstruction were remarkable, since the patients of the two clinical cases reported regained active extension of the hallux after 6 months without any associated complication. This study represents a step forward in foot surgery, since it describes an alternative technique to manage EHL tendon lesions.
\end{abstract}

\section{Introduction}

Lesions on the extensor hallucis longus (EHL) tendon are relatively rare and the few published studies concerning this injury provide unclear and conflicting treatment guidelines. ${ }^{1,2}$

The surgical management of acute EHL tendon lacerations may be relatively straightforward as long as the tendon edges are opposable. Secondary procedures motivated by chronic extensor apparatus impairment or failure of primary repairs, however, are more challenging and unpredictable. ${ }^{3}$ The purpose of this study was to report a series of two clinical cases treated with a combination of techniques for secondary EHL tendon reconstruction. Although the isolated techniques are already known, ${ }^{2,3}$ the combination of the two techniques has never been reported.

\section{Case Reports}

\section{Case 1}

A 23-year-old man presented with an inability to extend the hallux of the right foot ( - Fig. 1). One month earlier, the patient presented a EHL tendon laceration on zone 4 as a result of a crushing injury. The patient had undergone primary repair with a modified Kessler tenorrhaphy and epitenorrhaphy. However, the patient neglected the surgeons' instructions, removing the cast on the third postoperative day. The patient showed a complete inability to extend the interphalangeal joint of the hallux and 10 degrees of active metatarsophalangeal (MTP) joint extension. The flexion strength was normal and showed no limitation of articular MTP or interphalangeal (IF) excursion. The scar was excised, and local flaps were designed on the dorsum of the published online

November 30, 2020
DOI https://doi.org/

$10.1055 / \mathrm{s}-0040-1721525$ ISSN 0970-0358. (c) 2020. Association of Plastic Surgeons of India.

This is an open access article published by Thieme under the terms of the Creative Commons Attribution-NonDerivative-NonCommercial-License, permitting copying and reproduction so long as the original work is given appropriate credit. Contents may not be used for commercial purposes, or adapted, remixed, transformed or built upon. (https://creativecommons.org/licenses/by-nc-nd/4.0/)

Thieme Medical and Scientific Publishers Pvt. Ltd. A-12, 2nd Floor, Sector 2, Noida-201301 UP, India 


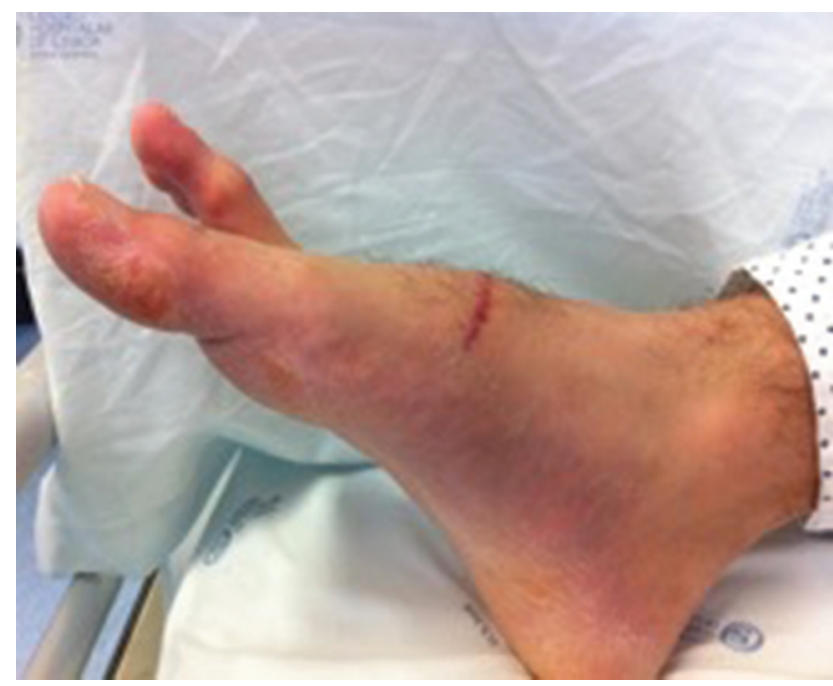

Fig. 1 Preoperative physical examination showing a complete inability to extend the hallux of the right foot.

foot, with careful placing of the resulting scars away from predictable tenorrhaphies. After raising the flap, the EHL tendon's severed ends were identified. The proximal end of the EHL tendon had retracted under the cruciate crural ligament ( - Fig. 2), and after tenolysis, a tendon gap of $3 \mathrm{~cm}$ remained. The patient had asked us to avoid other scars on the legs and arms; hence, we opted for an EHL elongation procedure (turnover) combined with a second-toe extensor tendon transfer. The procedure resulted in a figure of an "inverted Y," which was intraoperatively tested for excursion and passive strength ( - Fig. 3 ). The free distal end of the second-toe extensor tendon was sutured to the thirdtoe extensor tendon. The flaps were closed, and a short-leg cast was placed to attain an immobilization with the ankle at 90 degrees, and the MTP and IP joint at 15 and 0 degrees of extension, respectively.

A rehabilitation protocol was applied, and the patient began with controlled passive and active mobilization in the 5th postoperative week. ${ }^{4}$

\section{Case 2}

A 41-year-old woman, with an ankle arthrodesis in a context of a car accident some years earlier, presented with an inability to mobilize the hallux of the right foot ( - Fig. 4). The main problem was a mismatch between the flexor and extensor tension. The chronic pain, due to tendon adhesions, experienced when attempting to extend the hallux was the main complaint of this patient. Using MRI, we confirmed an entrapment of the extensor apparatus on the anterior border of the distal leg. The patient was indicated for tenolysis and EHL tendon reconstruction. Preoperative planning included flap design on the dorsum of the foot and markings over the

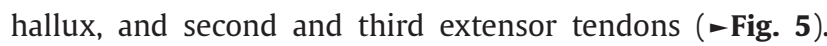
After raising the skin flaps, the peritendinous adhesions were released. After tenolysis, the EHL tendon showed insufficient length and a retracted position. Hence, we performed a Z-tenotomy, allowing for an overall tendon elongation

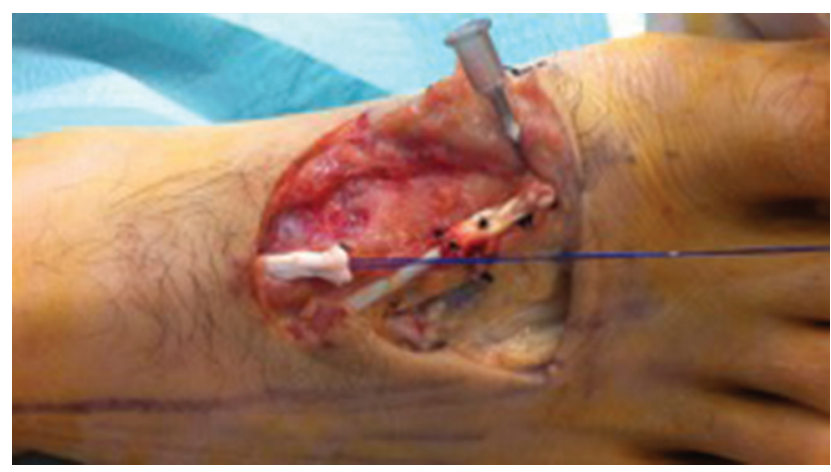

Fig. 2 Proximal end of the extensor hallucis longus (EHL) tendon retracted under the cruciate crural ligament.

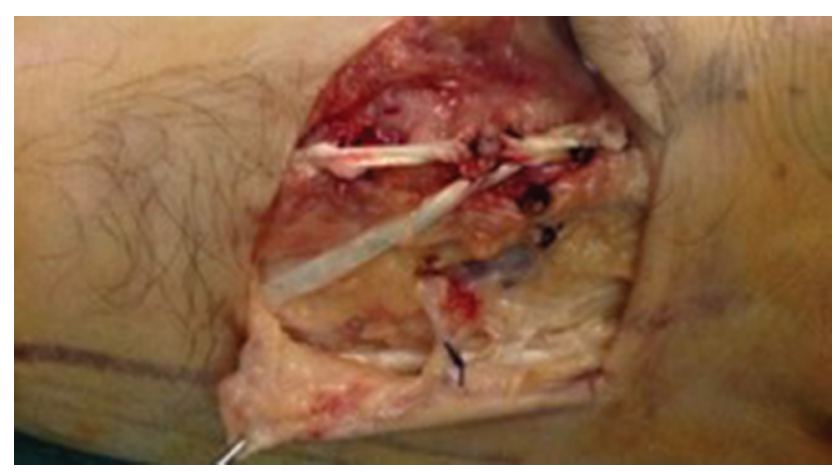

Fig. 3 A figure of "inverted $Y$ " resulting from the extensor hallucis longus (EHL) tendon elongation procedure (turnover) and second-toe extensor tendon transfer.

of $2.8 \mathrm{~cm}$ (-Fig. 6). Thereafter, the second-toe extensor was divided and the proximal stump was sutured to the EHL tendon. The free distal end of the second-toe extensor tendon was sutured to the third-toe extensor tendon, creating a figure of an inverted $Y(-$ Fig. 7). The hypertrophic scar on the distal leg, which resulted from ankle arthrodesis, was managed with autologous fat grafting. During the first 4 postoperative weeks, the patient remained nonweight-bearing and kept the foot immobilized in the short-leg cast. After that period, a rehabilitation program was initiated, with two therapy sessions per week. ${ }^{4}$

Both patients provided informed consent for this report.

\section{Discussion}

Our results show that a surgical approach with EHL tendon lengthening combined with second-toe extensor tendon transfer presents as a reliable and effective solution with excellent long-term functional results.

The current report refers to EHL tendon reconstruction in zone 4-dorsum of the foot. In this anatomical area, the most common injuries are osteochondral fractures, injury to dorsalis pedis artery, and transection of the terminal branch of the deep peroneal nerve. ${ }^{2,5}$ None of our patients had such comorbid injuries

In both patients, a primary repair was not an option, since the tendon edges were not opposable. Several tendon grafts or tendon transfer techniques are described in the literature; ${ }^{67}$ 


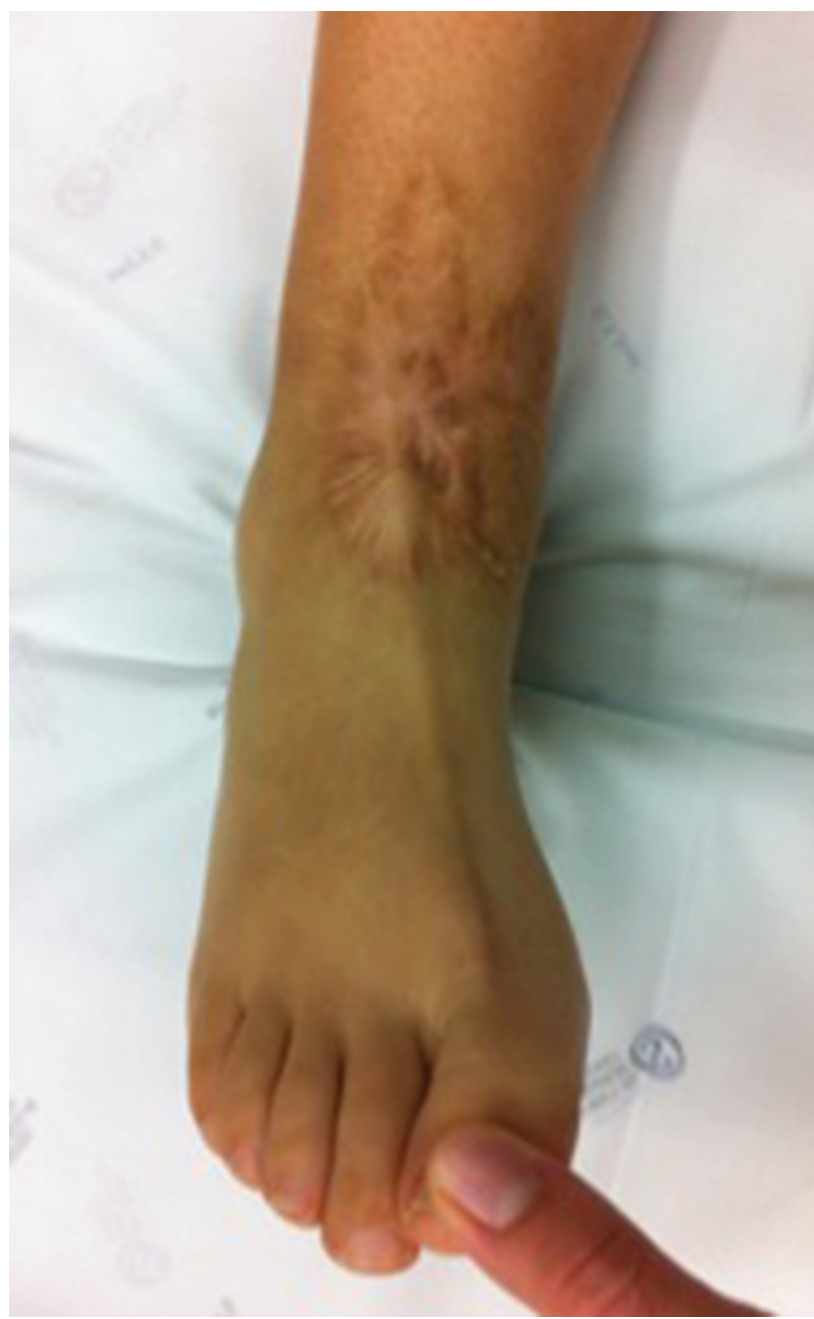

Fig. 4 Extensive scarring on the distal leg with subsequent entrapment of the extensor apparatus of the hallux. Note the extensor hallucis longus (EHL) tendon passing beneath the fibrotic tissue.

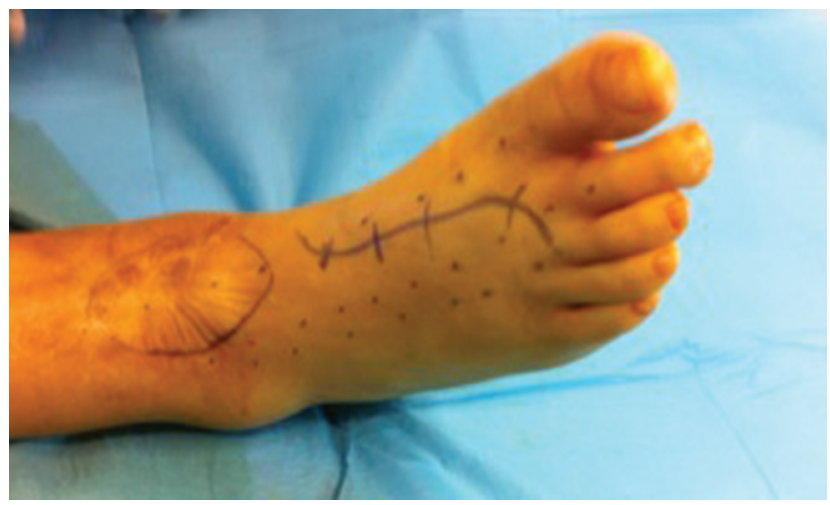

Fig. 5 Preoperative markings.

however, there is a scarcity of information about the delayed or secondary reconstruction of an EHL tendon. ${ }^{8}$ This is the first case report in which a combination of turnover elongation procedure and tendon transfer has been used for functional and anatomical restoration of the EHL tendon. To achieve a reliable reconstruction of the EHL tendon, the first

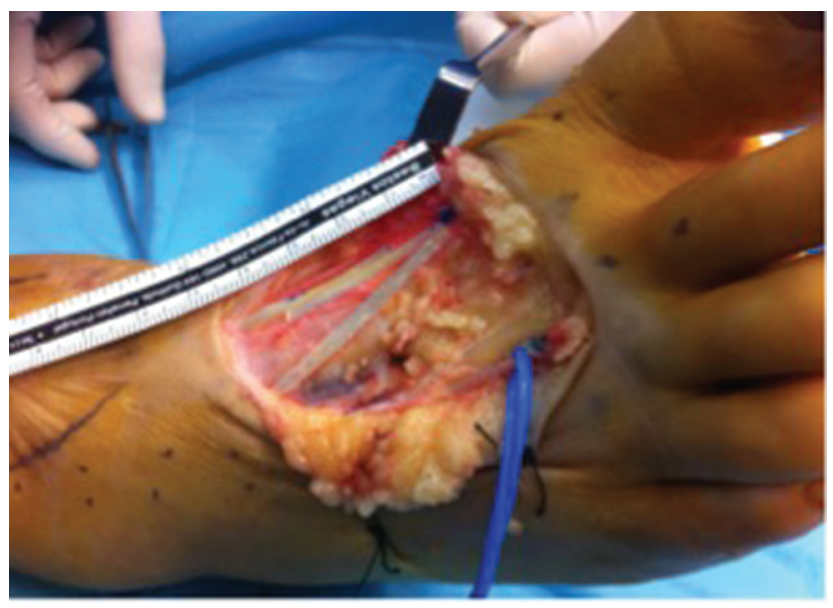

Fig. 6 Modified Z-tenotomy on zone 4 (dorsum of the foot), providing an overall tendon elongation of $2.8 \mathrm{~cm}$.

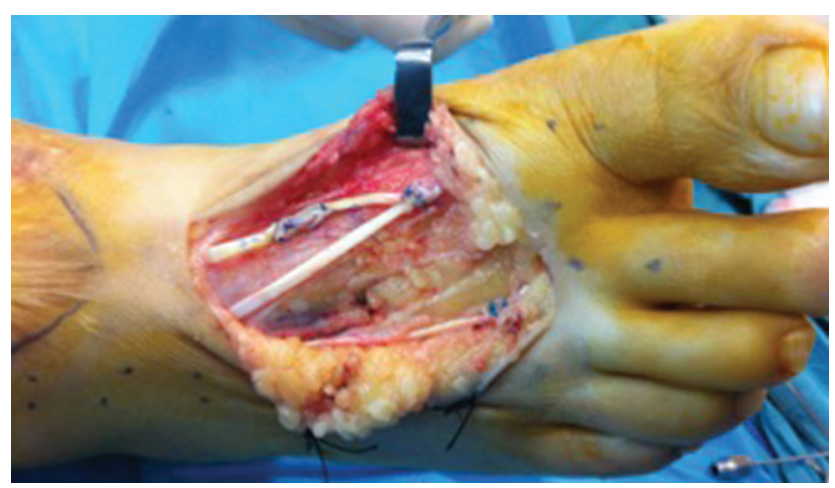

Fig. 7 A figure of "inverted $Y$ " resulting from the extensor hallucis longus (EHL) tendon elongation procedure (modified Z-tenotomy) and second-toe extensor tendon transfer.

step is to remove all fibrotic tissue around the tendon sheath to promote a normal gliding surface.

The tendon lengthening technique is a straightforward procedure indicated for tendon defects not exceeding 3 to $4 \mathrm{~cm}$, providing a means of immediate one-stage reconstruction with no secondary site morbidity and the advantage of not compromising any further surgical repair. ${ }^{9}$ The tendon lengthening may cause some degree of tendon weakness in a tendon that may be already damaged because of trauma or prior surgical interventions. This was the case for both patients, leading to a greater chance of an eventual rupture or functional limitations during extension of the hallux. Hence, there is no doubt that additional tendon transfer must be performed to achieve a more reliable tendon repair. The choice for the second-toe extensor tendon and avoidance of other potential donor sites is justified by anatomic adjacency, easy dissection, and no donor site morbidity. ${ }^{10}$

The functional results as per the American Orthopaedic Foot \& Ankle Society (AOFAS) hallux scale were very good in the first case, with 45 points out of 45 and 35 out of 45 points for the second patient. Both patients presented with normal range of motion of the hallux 6 months after the surgery, with the results graded as good according to Lipscomb and Kelly. Additionally, both patients were free of pain 6 months after 
the surgical intervention. The lower score for the second case may be explained by the complex orthopedic background of this patient and is most likely not due to the different techniques in each case.

\section{Conclusions}

We present a surgical approach for secondary injuries of the EHL tendon by combining tendon lengthening with second-toe extensor transfer. This technique has proved to be a reliable and effective solution with excellent long-term functional results.

\section{Conflicts of Interest}

None declared.

\section{References}

1 Poggi JJ, Hall RL. Acute rupture of the extensor hallucis longus tendon. Foot Ankle Int 1995;16(1):41-43

2 Joseph RM, Barhorst J. Surgical reconstruction and mobilization therapy for a retracted extensor hallucis longus laceration and tendon defect repaired by split extensor hallucis longus tendon lengthening and dermal scaffold augmentation. J Foot Ankle Surg 2012;51(4):509-516

3 Wong JC, Daniel JN, Raikin SM. Repair of acute extensor hallucis longus tendon injuries: a retrospective review. Foot Ankle Spec 2014;7(1):45-51

4 Mowlavi A, Schall J, Wilhelmi BJ. Extensor hallucis longus tenorrhaphy by using the Massachusetts General Hospital repair. J Foot Ankle Surg 2004;43(6):412-418

5 Thordarson DB, Shean CJ. Nerve and tendon lacerations about the foot and ankle. J Am Acad Orthop Surg 2005;13(3):186-196

6 Park HG, Lee BK, Sim JA. Autogenous graft repair using semitendinous tendon for a chronic multifocal rupture of the extensor hallucis longus tendon: a case report. Foot Ankle Int 2003;24(6):506-508

7 Smith B, Coughlin M. Reconstruction of a chronic extensor hallucis longus tendon laceration with a gracilis tendon autograft. Orthopedics 2008;31(10):31

8 Al-Qattan MM. Surgical treatment and results in 17 cases of open lacerations of the extensor hallucis longus tendon. J Plast Reconstr Aesthet Surg 2007;60(4):360-367

9 Cerovac S, Miranda BH. Tendon 'turnover lengthening' technique. J Plast Reconstr Aesthet Surg 2013;66(11):1587-1590

10 Leung YF, Ip SP, Chung OM. A new method of functional tendon transfer for the dysfunction of extensor hallucis longus. Foot Ankle Int 2002;23(12):1124-1125 